\title{
Research on Targeted Thermal Effect of Molybdenum Disulfide Nanosheets Modified by Nucleic Acid Aptamers
}

\author{
Bo pang, Lihong Jin*, Liying Wang \\ China School of Life Science and Technology, Changchun University of Science and Technology, Changchun, China
}

\begin{abstract}
In this paper, the surface of molybdenum disulfide nanosheets synthesized by hydrothermal method was modified with bovine serum albumin (BSA) and nucleic acid aptamers in order to construct a photothermal system with high target recognition, stable size, good biocompatibility and low toxicity Nano material molybdenum disulfide; Under $808 \mathrm{~nm}$ laser irradiation, suitable functionalized molybdenum disulfide nanosheets can produce singlet oxygen at an effective concentration for photodynamic therapy, and at the same time, photothermal therapy can also be used to achieve synergistic therapy and the therapeutic effect is more excellent. This shows that such functionalized molybdenum disulfide nanosheets have excellent therapeutic effect and great potential in tumor treatment.
\end{abstract}

\section{Introduction}

Cancer is one of the diseases with the highest morbidity and mortality in the world, and photothermal therapy is a new type of treatment [1-4]. At present, the widely studied photothermal materials mainly include precious metal nanoparticles, organic nanophotothermal conversion materials, carbon-based photothermal conversion materials. [5-7] Molybdenum disulfide has costs Low cost, good stability, excellent light and heat absorption and other advantages, and show higher absorbance in the NIR region than graphene and gold nanorods [8]. At present, many studies are more inclined to the study of functionalized molybdenum disulfide. However, the large-scale multifunctional molybdenum disulfide is not targeted and cannot be enriched at the tumor site to achieve accurate and efficient treatment. Therefore, this article will develop a targeted functionalized molybdenum disulfide nanosheet that can be highly specific Molybdenum disulfide nanosheets are effectively enriched in tumor tissue to improve the treatment efficiency.

The nucleic acid aptamer refers to a type of oligonucleotide sequence that can specifically bind to the target substance and can specifically identify tumor cells. Based on this, this article will use the hydrothermal method and the assisted ultrasonic stripping method to construct the $\mathrm{MoS}_{2}$-BSA nanometer.After the aptamer was coupled to $\mathrm{MoS}_{2}$-BSA, $\mathrm{MoS}_{2}$-BSA withsmall size, temperature-temperature reduction, suspension stability, and near-infrared absorption characteristics and good photothermal temperature rise effect was prepared. $\mathrm{MoS}_{2}-$ BSA-Aptnanosheets. $\mathrm{MoS}_{2}$-BSA-Apt nanosheets can specifically recognize target cells and be transported into the cells. The photothermal treatment process is accompanied by singlet oxygen production and damage to cellular mitochondria, which in turn causes rapid apoptosis of tumor cells; Experiments have proved that $\mathrm{MoS}_{2}$-BSA-Apt nanosheets have specific recognition of target cells, low toxicity, good biocompatibility and photothermal properties, and they are expected to be used in tumor precise treatment.

\section{Preparation of Functionalized $\mathrm{MoS}_{2}$ Nanosheets}

\subsection{Glucose-assisted synthesis of molybdenum disulfide nanosheets}

Add the L-cysteine solution and a certain amount of stripping agent glucose to the sodium molybdate solution in sequence, then sonicate for $10 \mathrm{~min}$, and then hydrothermal Reaction, centrifugation to obtain molybdenum disulfide nanosheets; Among them, glucoseassisted one-step hydrothermal synthesis of $\mathrm{MoS}_{2}$ nanosheets, in which the mass ratio of sodium molybdate to L-cysteine is 1: 2 . Whether to add glucose stripping agent $(6.25 \mathrm{mg}, 62.5 \mathrm{mg})$ and reaction time (10h, 24h, 36h) were used as synthesis condition variables.

\subsection{Surface Modification of Bovine Serum Albumin with $\mathrm{MoS}_{2}$ Nanosheets}

After mixing the synthesized $\mathrm{MoS}_{2}$ nanosheets and BSA in the solution at a mass ratio of $3: 1$, stirring in an ice bath or room temperature for $3 \mathrm{~h}$, and centrifuging at $4^{\circ} \mathrm{C}$ and $12000 \mathrm{rpm}$ for $30 \mathrm{~min}$ to obtain surface-modified bovine serum albumin II Molybdenum sulfide nanosheets, in which the ice bath ultrasonic time $(2 \mathrm{~h}, 4 \mathrm{~h})$ and frequency

\footnotetext{
*Corresponding author: 825125176@qq.com
} 
$(25 \mathrm{HZ}, 45 \mathrm{HZ})$ were stirred at room temperature for $3 \mathrm{~h}$ as synthesis condition variables.

\section{3. $\mathrm{MoS}_{2}$-BSA ligated aptamer}

EDC and NHS are used to activate the free carboxyl groups at the end of the surface of $\mathrm{MoS}_{2}-\mathrm{BSA}$, and then washed by ultrafiltration centrifugal deionized water to obtain activated nanoparticles; Denature the nucleic acid aptamer at $85^{\circ} \mathrm{C}$ for $10 \mathrm{~min}$ and then renature in ice bath for $10 \mathrm{~min}$; The activated $\mathrm{MoS}_{2}$-BSA sheet was reacted with nucleic acid aptamer Apt1 (herein abbreviated as Apt) for $5 \mathrm{~h}$, and then centrifuged by ultrafiltration until no aptamer was detected in the ultrafiltrate, and finally the aptamer-modified $\mathrm{MoS}_{2}$ - BSA-Apt was obtained.

\section{Determination of photothermal effect of functionalized molybdenum disulfide nanosheets}

\subsection{MoS $_{2}-B S A$ photothermal effect}

Irradiate $0.2 \mathrm{mg} \cdot \mathrm{mL}^{-1} \mathrm{MoS}_{2}$-BSA aqueous solution with NIR laser $\left(808 \mathrm{~nm}, 0.8 \mathrm{~W} \cdot \mathrm{cm}^{-2}\right)$ for 3 minutes, during which the temperature changes were recorded by an infrared thermal imager with a recording time interval of $20 \mathrm{~s}$, Plot the temperature rise curve of $\mathrm{MoS}_{2}-\mathrm{BSA}$.

\subsection{Photothermal effect of MoS $_{2}-B S A-A p t$ solutions with different concentrations}

The concentrations of $0.025,0.05,0.1$ and $0.2 \mathrm{mg} \cdot \mathrm{mL}^{-}$ ${ }^{1} \mathrm{MoS}_{2}$-BSA-Apt solutions were irradiated with laser respectively, and the temperature conditions were recorded and plotted. The laser parameters are the same as before.

\subsection{Characterization of Functionalized $\mathrm{MoS}_{2}$ Nanosheets}

In the experiment, it was characterized by transmission electron microscopy, and then X-ray diffraction energy spectrum was used to determine the crystal structure of the related materials of $\mathrm{MoS}_{2}$ nanosheets. The ultravioletvisible-near infrared absorption spectrometer was used to characterize the successful modification of the surface of $\mathrm{MoS}_{2}$ nanosheets. Load BSA and nucleic acid aptamers.

\section{Cytotoxicity of functionalized molybdenum disulfide nanoparticles and photothermal properties of tumor cells in vitro}

\subsection{Fluorescence inverted microscope characterization}

Take MCF-7 and MCF-10A cell add $0.1 \mathrm{mg} \cdot \mathrm{mL}^{-1}$ of $\mathrm{MoS}_{2}$-BSA-Apt2 (Apt2 is modified with a fluorophore) solution. After incubation on ice, wash the cells with PBS. Observe MCF-7 human breast cancer cells and MCF-10A human breast cells with a fluorescent inverted microscope.

\subsection{In vitro cytotoxicity test}

Cultivate MCF-7 human breast cancer cells, add culture medium containing $\mathrm{MoS}_{2}$-BSA with different concentrations. Put in a $\mathrm{CO}_{2}$ incubator and incubate, then add MTT solution, continue the culture for $4 \mathrm{~h}$, aspirate the culture solution, and then add DMSO. Under dark conditions, transfer to a microplate reader to measure its absorbance and record the test data.

\subsection{In vitro cell photothermal therapy experiment}

The same batch of MCF-7 human breast cancer cells were seeded on 96-well plates and cultured until the cells adhered. Cells were randomly divided into 8 groups, control group, laser irradiation only group, $\mathrm{MoS}_{2}$-BSA only culture group, $\mathrm{MoS}_{2}$-BSA-Apt only culture group, $\mathrm{MoS}_{2}$-BSA plus laser irradiation for 5 min group, material plus laser irradiation for $10 \mathrm{~min}$ group. $\mathrm{MoS}_{2}$-BSAnucleic acid aptamer plus laser irradiation for 5 min group, materials plus laser irradiation for $10 \mathrm{~min}$ group. After the cells adhere to the wall, add $0.1 \mathrm{mg} \cdot \mathrm{mL}^{-1} \mathrm{MoS}_{2}$-BSA nanosheet and $0.1 \mathrm{mg} \cdot \mathrm{mL}^{-1} \mathrm{MoS}_{2}$-BSA-Apt nanosheet solution prepared with culture solution and materials, and continue incubating in the incubator. After $12 \mathrm{~h}$, the particles are allowed to enter the cell. After $12 \mathrm{~h}$, the solution was sucked out, washed with PBS, 200 $\mu$ l of culture solution was added to the laser irradiation group and then irradiated with NIR laser $\left(808 \mathrm{~nm}, 0.8 \mathrm{~W} \cdot \mathrm{cm}^{-2}\right)$, washed with PBS, and added with calcein-PI dye to continue incubation for $20 \mathrm{~min}$ after aspirating and washing with PBS, cell fluorescence information was observed under a fluorescence microscope.

\section{Results and discussion}

\subsection{Photothermal effect of functionalized molybdenum disulfide nanosheets}

Fig.1(a) shows that the $\mathrm{MoS}_{2}$-BSA nanosheet solutions obtained under different experimental conditions have a certain temperature increase after being irradiated with laser, Among them, the highest temperature rise was $63.18^{\circ} \mathrm{C}$, and the temperature increase was $43.18^{\circ} \mathrm{C}$. The results show that the photothermal effect of $\mathrm{MoS}_{2}$-BSA obtained under hydrothermal synthesis and construction conditions is the largest at $2 / 24 / 45 / 2 / 0$ (identification numbers represent glucose addition / hydrothermal time / ultrasonic frequency / ultrasonic time / stirring time, respectively). As shown in Fig.1(b), the temperature of $\mathrm{MoS}_{2}$-BSA-Apt solutions with different concentrations all increase with the irradiation time. The higher the concentration, the faster the temperature rise, the greater the temperature increase, and the higher the increase. In the control group, and meet the temperature requirements of photothermal treatment. 

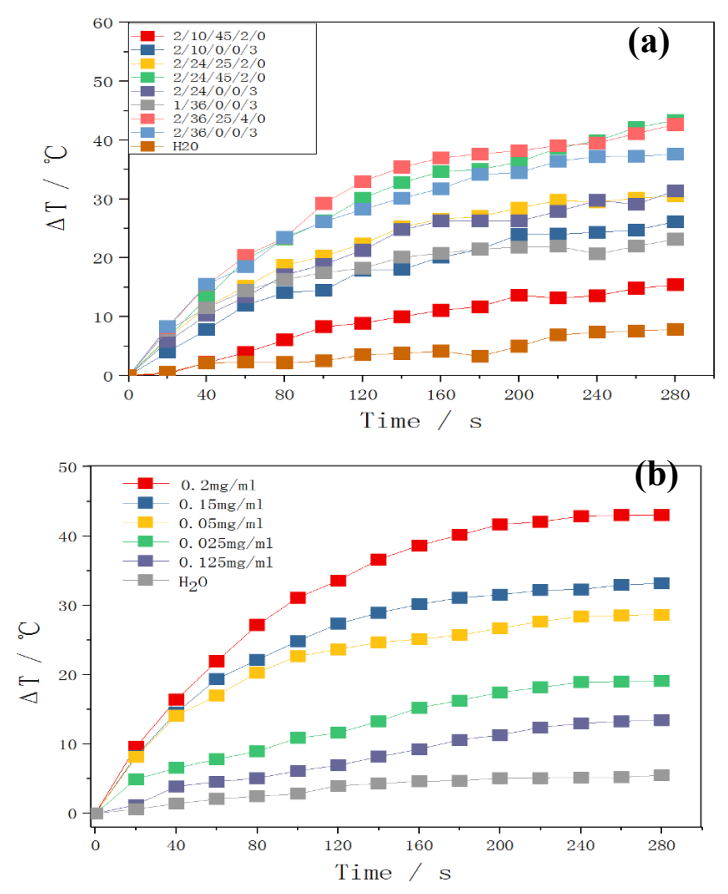

Fig. 1. (a) $\mathrm{MoS}_{2}$-BSA temperature rise curve obtained by different synthesis and construction conditions (variable group with larger temperature rise) (b) Photothermal effect of different concentrations of $\mathrm{MoS}_{2}$-BSA-Apt

\subsection{Characterization of Functionalized $\mathrm{MoS}_{2}$ Nanosheets}

From TEM image 2(a), it can be seen that the morphology of $\mathrm{MoS}_{2}$-BSA-Apt nanometer tablets is not strong, and the size is in the range of $100 \mathrm{~nm}-150 \mathrm{~nm}$, which is a layer structure.

X-ray diffraction diagram 2(b) shows compared with the JCPDS standard card (No.73-1508), it can be seen that the $40^{\circ}$ corresponds to the molybdenum disulfide (103) crystal plane, and the $58^{\circ}$ corresponds to the molybdenum disulfide (110) crystal plane. From this, $\mathrm{MoS}_{2}$ and BSA, BSA-Apt The molybdenum disulfide phase was not changed after the connection, and the functionalized molybdenum disulfide nanosheet product was hexagonal phase molybdenum disulfide.

As can be seen in Fig. 2(c) and (d), the $\mathrm{MoS}_{2}$ nanosheet solution has light absorption characteristics in the range of 200-1100 nm, and its maximum absorption peak is at 210 $\mathrm{nm}$; while the maximum absorption peak of BSA is at 280 $\mathrm{nm}, \mathrm{MoS}_{2}$-BSA The solution also had an absorption peak at $280 \mathrm{~nm}$, indicating that BSA was successfully wrapped on the surface of $\mathrm{MoS}_{2}$ nanosheets.In addition, the UV absorption peak of DNA at $260 \mathrm{~nm}$ is caused by the baseconjugated double bond. After coupling the nucleic acid aptamer to the $\mathrm{MoS}_{2}$-BSA nanosheet, no BSA and DNA absorption peaks were detected in the supernatant. It shows that Apt is coupled to $\mathrm{MoS}_{2}$-BSA nanosheets; and $\mathrm{MoS}_{2}$-BSA-Apt has a characteristic peak at $263 \mathrm{~nm}$, which confirms that the nucleic acid aptamer is successfully coupled to the surface of $\mathrm{MoS}_{2}$-BSA.
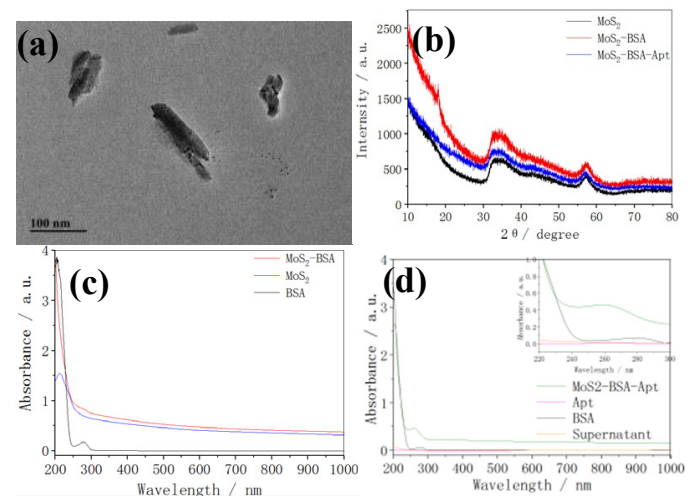

Fig. 2. (a) Transmission electron micrograph of $\mathrm{MoS}_{2}-\mathrm{BSA}-\mathrm{Apt}$ nanosheets (b) $\mathrm{MoS}_{2}, \mathrm{MoS}_{2}-\mathrm{BSA}$ and $\mathrm{MoS}_{2}-\mathrm{BSA}-\mathrm{Apt}$ nanosheets XRD (c) $\mathrm{MoS}_{2}, \mathrm{MoS}_{2}$-BSA and BSA UV-Vis-NIR Absorption spectrum; (d) $\mathrm{MoS}_{2}$-BSA-Apt, Apt, BSA and supernatant ultraviolet-visible-near infrared absorption spectrum

\subsection{Targeted detection and cytotoxicity of functionalized molybdenum disulfide nanosheets}

Fig.3 (a)shows, MoS $_{2}$-BSA-Apt has excellent fluorescence recognition ability to target tumor cells. As shown in Fig.3 (b), the cell survival rate gradually decreases with the increase of the concentration of the nanosheet solution. When the concentration is $200 \mu \mathrm{g} \mathrm{mL}-$ 1 , the cell survival rate is not less than $75 \%$ is in line with the conditions of photothermal therapy.
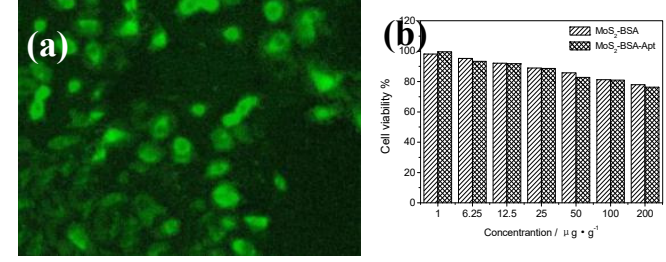

Fig. 3. (a) $\mathrm{MoS}_{2}-\mathrm{BSA}-\mathrm{Apt}$ incubating $\mathrm{MCF}-7$ human breast cancer cells; (b) $\mathrm{MoS}_{2}$-BSA and $\mathrm{MoS}_{2}$-BSA-Apt cytotoxicity detection

\subsection{In vitro cell photothermal therapy of functionalized molybdenum disulfide nanosheets}

Fig. 4(a) (h)shows: It can be seen that the cells in groups (a), (b), (c), and (d) have almost no death, indicating that $\mathrm{MoS}_{2}$-BSA-Apt and $\mathrm{MoS}_{2}$-BSA nanosheets have no damage to MCF-7 human breast cancer cells at this concentration And the laser of this power density will not cause damage to the cells. After culturing the cells with two molybdenum disulfide nanosheet solutions, NIR irradiation was performed for 5 minutes, and after 10 minutes, they showed different degrees of death. The number of cell death increased with the increase of laser irradiation time; Comparing the two groups of nanosheet treatment groups with the same irradiation time, it was found that the number of cell deaths in the $\mathrm{MoS}_{2}-\mathrm{BSA}-\mathrm{Apt}$ nanosheet solution group was more than the $\mathrm{MoS}_{2}$-BSA nanosheet solution group, indicating that $\mathrm{MoS}_{2}-\mathrm{BSA}$ and $\mathrm{MoS}_{2}$-BSA-Apt nanosheet solution All have good photothermal effects. $\mathrm{MoS}_{2}$-BSA-Apt nanosheets have 
better ability to identify MCF-7 human breast cancer cells, so the photothermal treatment effect of $\mathrm{MoS}_{2}-\mathrm{BSA}-\mathrm{Apt}$ is better than $\mathrm{MoS}_{2}$-BSA nanosheets.

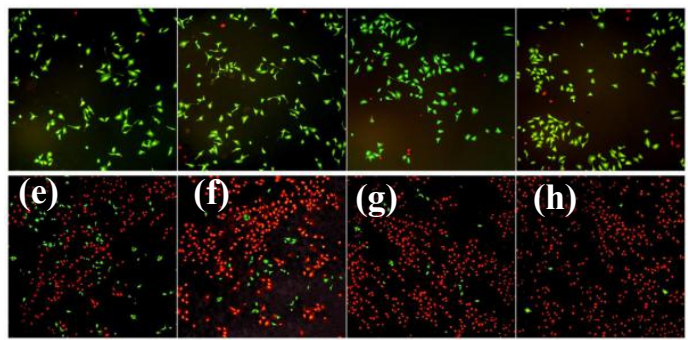

Fig. 4. Photographs of fluorescent staining for cancer cell phototherapy in vitro (a) Control group; (b) $\mathrm{MoS}_{2}-\mathrm{BSA}-\mathrm{Apt}$ only; (c) $\mathrm{MoS}_{2}$-BSA only; (d) $808 \mathrm{~nm}$ near infrared laser irradiation only; (e) $\mathrm{MoS}_{2}-\mathrm{BSA}+5$ min NIR; (f) $\mathrm{MoS}_{2}-\mathrm{BSA}-\mathrm{Apt}+5$ min NIR; (g) $\mathrm{MoS}_{2}$-BSA + 10 min NIR; (h) $\mathrm{MoS}_{2}$-BSA- Apt +10 $\min$ NIR

\section{References}

1. S.P. Zhang, Y.X. Cheng, L. Ren, et al. Synthesis and photothermal properties of Prussian blue nanoparticles with different shapes. Chemical Journal of Chinese Universities, 2018, vol.39, no.2, pp.359366.

2. D. Zhang, F.F. Zhou, D. Xing, Targeted tumor imaging and photothermal treatment of functionalized graphene oxide. Science Bulletin, 2013, no.7, pp.586-592.

3. W.S. Xie, Q. Gao, Z.H. Guo, et al. Research progress of functional composite biomaterials for tumor local hyperthermia. Modern Oncology, 2018, vol.26, no.3, pp.474-477.

4. F. Zhou, G.Y. Deng, X.J. Liu, et al. Cytotoxicity and photothermal stability of new copper sulfide nanoparticles. Chinese Journal of Experimental Surgery, 2017, vol.34, no.11, pp.1875-1879.

5. T.F. Jiao, X.X. Huang, L.X. Zhang, et al. Progress in synthesis and application of photothermal / photosensitizer nanomaterials. Journal of Yanshan University, 2017, vol.41, no.3, pp.189-203.

6. Y.S. Li, N.B. Zhong, Q. Liao, et al. Photothermal conversion biomaterials based on lanthanum hexaboride and chitosan. Acta Luminica Sinica, 2017, vol.38, no.8, pp.1021-1027.

7. X.Y. Li, M.W. Ji, H.Z. Wang, et al. Research progress of near-infrared photothermal conversion nanocrystals. China Optics, 2017, vol.10, no.10, pp.541-554.

8. Y.H. Li, Anti-human ADAM15 antibody inhibits tumor proliferation and tumor targeting experiment. Suzhou University, 2012. 